\title{
Evaluation of the Effects of COVID-19 Pandemic Among Patients with Inflammatory Bowel Diseases
}

\author{
(1) Yasemin Gökden¹, (1) Nergiz Ekmen², (1) Mine Adaş3, (1) Süheyla Atak33, (1) Hadi Sasani4 \\ 1 University of Health Sciences Turkey, Prof. Dr. Cemil Taşçıŏlu City Hospital, Clinic of Gastroenterology, İstanbul, Turkey \\ 2 Gazi University Faculty of Medicine, Department of Gastroenterology, Ankara, Turkey \\ 3 University of Health Sciences Turkey, Prof. Dr. Cemil Taşçıoğlu City Hospital, Clinic of Internal Medicine, İstanbul, Turkey \\ ${ }^{4}$ Tekirdağ Namık Kemal University Faculty of Medicine, Department of Radiology, Tekirdağ, Turkey
}

\section{Abstract}

Objective: The Coronavirus Disease-2019 (COVID-19) pandemic continues to be a concern, especially to people with chronic diseases across the entire world. During this pandemic, we undertook an investigation to assess how the disease state and medical treatments of inflammatory bowel disease (IBD) are affecting the IBD patients, their risky perceptions, as well as the frequency and course of COVID-19.

Methods: During the pandemic, the information on the course of the disease, medical treatment status of the patients with IBD, and the course of the disease in IBD patients who had COVID-19 were collected via telephonic interview.

Results: A total of 102 IBD patients, including 62 with ulcerative colitis and 40 with Crohn's disease were included in the study. Of these, $52.9 \%$ of the patients believed that having IBD was a risk for COVID-19. During the pandemic, 18.6\% of the patients did not take their medication regularly for various reasons. Of all, $64.28 \%$ of the patients with active disease could not go to the hospital to avoid the risk of acquiring COVID-19 and 4.90\% acquired COVID-19.

Conclusion: During the pandemic, both the regular consumption of medicines and the reservations regarding admission to hospitals affect the course of IBD. Therefore, the development of strategic action plans to support and manage changes experienced during the course of this disease is expected to facilitate the management of the disease process in a healthier way for both the physicians and the patients.

Keywords: Inflammatory bowel disease, COVID-19, ulcerative colitis, Crohn disease

\section{INTRODUCTION}

Since the first cases were detected in Wuhan, China in December 2019, the new Coronavirus Disease-2019 (COVID-19) Severe Acute Respiratory syndrome-coronavirus-2 (SARS-CoV-2) has affected the entire world population, creating a serious public health issue. Although, initially, it was believed to be a pure respiratory pathogen transmitted via droplets and aerosols, it has also now been observed to cause severe symptoms of the gastrointestinal system (1). The most common symptoms of COVID-19 are fever and respiratory distress. However, a significant proportion of COVID-19 patients also have digestive system symptoms such as changes in their bowel habits, diarrhea, vomiting, and anorexia (2). SARS-CoV-2 enters the cells via angiotensin-converting enzyme 2 (ACE2) receptor (3). The ACE2 receptor is expressed in several organs, such as the lung, tongue, pancreas, and intestines (4). Gastrointestinal symptoms (GIS) are caused by the ACE2 expression in the intestine (5).

Ulcerative colitis (UC) and Crohn's disease (CD) are the two major forms of inflammatory bowel diseases (IBDs) and are characterized by remission and exacerbation episodes (6). It is understandable that patients with IBD experience weakness and are worried about acquiring COVID-19 infection during the 
pandemic period due to the nature of the disease transmission and the immunomodulatory drugs used in the treatment process.

SARS-CoV-2 has spread rapidly to the entire world from China, after which the World Health Organization declared the COVID-19 pandemic on March 11, 2020. As in almost all countries of the world, extremely serious and strict public health regulations and strict quarantine measures have been taken in order to prevent the further spread of the disease as well as to provide the treatment to patients diagnosed with COVID-19. During this process, it has become difficult for patients with chronic diseases, such as IBD patients, to manage their medical follow-up and treatment. In addition, the combat against COVID-19 has gained priority among all healthcare professionals at all levels, which has made it difficult for non-COVID-19 patients to reach health institutions for treatment. Patients with IBD may need additional endoscopic procedures and several more potent medical treatments, especially during the exacerbation periods. However, as in several countries, routine endoscopic procedures were temporarily suspended in Turkey with the recommendations of health authorities and only emergency endoscopic procedures. This situation may have resulted in a delay in the routine planned procedures, and more effective treatments plans had to be initiated in case of disease exacerbation. Due to the ensuing concerns about the risk of COVID-19 transmission on their illnesses and medications, IBD patients are likely to face difficulties in accessing the clinical centers and physicians for follow-up. In addition, some patients may have discontinued or postponed their medical treatment during this period.

In this study, we aimed to investigate the extent to which the disease status and medical treatments of IBD patients were affected during the COVID-19 pandemic, as well as their perception about infection risk and the frequency and course of COVID-19.

\section{METHODS}

This study was approved by the Prof. Dr. Cemil Tașçıŏglu City Hospital/Local Human Research Ethics Committee, and all procedures conducted in the study involving human participants were in accordance with the ethical standards of the institutional and/or national research committee and with the 1964 Helsinki Declaration and its later amendments or comparable ethical standards. This study was approved by the Local Ethics Committee (12/05/2020 decision no: 156).

All subjects included in this study agreed to their participation with their informed consent for the processing and collection of data for scientific purposes. A detailed questionnaire was administered to all subjects by telephone or email.

\section{Patients Selection}

Patients with follow-up medical files from the gastroenterology outpatient clinical department of the institution, and who were aged $>18$ years and were clinically, endoscopically, radiologically, and histopathologically diagnosed with IBD (UC or CD) were included in the study.

Patients with an uncertain diagnosis of IBD, age $<18$ years, with incomplete information in their medical files, and those who could not be contacted via phone or who did not agree to participate in the study or who had incomplete answers to the questions were excluded from the study. Finally, 102 (71.83\%) of the 142 patients who were followed up with UC and CD were included for study analysis because they met the inclusion criteria (Table 1).

The subjects were asked whether there was an exacerbation related to IBD diseases in the 3 month period from March 15, 2020 to June 15, 2020, when COVID-19 cases are detected in our country, about their endoscopy needs and accessibility to the hospital, changes in their medical treatments and the affecting parameters, the other diseases and the medical treatments used; the patient perception about the risk to COVID-19 infection in terms of their illnesses and medical treatments, and the diseaserelated parameters (disease involvement site) and whether they were diagnosed with COVID-19 during this period, their requests for follow-up, symptoms of those diagnosed with COVID-19, and the course of the disease and the management of the drugs were questioned.

Harvey Bradshaw for CD patients and the partial Mayo score for UC were applied to assess the disease activity of the subjects.

\section{Statistical Analysis}

Data were analyzed using the statistical package program (SPSS 20.0). The mean \pm standard deviation and median for descriptive data and continuous variables and the numbers and percentages for categorical variables were presented. ShapiroWilk test was applied to evaluate the normal distribution of the data. In the analysis of the changes within the data, the ones selected according to the distribution characteristics, Pearson's chi-square test, Fisher exact chi-square test, McNemar test, Wilcoxon test, and t-test in dependent groups were used. During the evaluation of the results, $p \leq 0.05$ was considered to be statistically significant. 


\section{RESULTS}

A total of 102 IBD patients [62 (60.8\%) UC and 40 (39.2\%) CD] were included in the study. The mean age of the UC group was $40.87 \pm 13.81$ years and that of the CD group was $40.20 \pm 12.75$

\begin{tabular}{|c|c|c|}
\hline & $C D(n=40) n(\%)$ & $U C(n=62) n(\%)$ \\
\hline \multicolumn{3}{|l|}{ Age } \\
\hline$\leq 24$ & $6(15)$ & 8 (12.9) \\
\hline $25-34$ & $5(12.5)$ & $14(22.6)$ \\
\hline $35-44$ & $16(40)$ & $15(24.2)$ \\
\hline $45-54$ & $9(22.5)$ & $13(21.0)$ \\
\hline $55-64$ & $3(7.5)$ & $10(16.1)$ \\
\hline$\geq 65$ & $1(2.5)$ & $2(3.2)$ \\
\hline \multicolumn{3}{|l|}{ Gender } \\
\hline Female & $23(57.5)$ & $28(45.2)$ \\
\hline Male & $17(42.5)$ & $34(54.8)$ \\
\hline \multicolumn{3}{|l|}{ Occupation } \\
\hline Unemployed & $3(7.5)$ & $0(0.0)$ \\
\hline Retired & $3(7.5)$ & $10(16.1)$ \\
\hline Housewife & $13(32.5)$ & $16(25.8)$ \\
\hline Self-employment & $4(10)$ & $4(6.5)$ \\
\hline Other & $17(42.5)$ & $32(51.6)$ \\
\hline \multicolumn{3}{|l|}{ Marital status } \\
\hline Married & $30(75)$ & $45(72.6)$ \\
\hline Single & $10(25)$ & $17(27.4)$ \\
\hline \multicolumn{3}{|l|}{ Education status } \\
\hline Primary education & $20(50)$ & $30(48.4)$ \\
\hline High school & $16(40)$ & $20(32.3)$ \\
\hline University & $4(10)$ & $12(19.4)$ \\
\hline \multicolumn{3}{|l|}{ Previous operation } \\
\hline Yes & $8(20)$ & $3(4.8)$ \\
\hline No & $32(80)$ & $59(95.2)$ \\
\hline \multicolumn{3}{|l|}{ Age at diagnosis } \\
\hline$\leq 24$ & $9(22.5)$ & $14(22.6)$ \\
\hline $25-34$ & $13(32.5)$ & $20(32.3)$ \\
\hline $35-44$ & $11(27.5)$ & $13(21.0)$ \\
\hline $45-54$ & $5(12.5)$ & $9(14.5)$ \\
\hline $55-64$ & $1(2.5)$ & $5(8.1)$ \\
\hline$\geq 65$ & $1(2.5)$ & $1(1.6)$ \\
\hline \multicolumn{3}{|l|}{ Duration of disease } \\
\hline $3 \leq$ & $14(35.0)$ & $29(46.8)$ \\
\hline $4-7$ & $17(42.5)$ & $16(25.8)$ \\
\hline 8-11 & $2(5.0)$ & $4(6.5)$ \\
\hline $12 \geq$ & $7(17.5)$ & $13(21.0)$ \\
\hline
\end{tabular}

years; the disease duration of $U C$ and $C D$ was $6.29 \pm 6.06$ and $6.58 \pm 6.70$ years, respectively. The demographic data are shown in Table 1.

Comparison of the data about the patients' weight and body mass index (BMI) before and during the pandemic revealed no statistically significant difference in the UC groups, but both the weight and BMI in the CD group had increased during the pandemic than before it ( $p=0.010, p=0.022$, respectively).

When IBD was considered as a single group and the patients were questioned whether they consider the risk of acquiring COVID-19 as a high risk due to their disease, 54 (52.9\%) of the patients believed that IBD was a risk for COVID-19 (Table 2). The age, gender, year of illness, age of diagnosis, marital status, educational status, and surgery status did not have a statistically significant difference in terms of perception about the risk of acquiring COVID-19 as a high risk. In addition, when questioned whether the drugs used could increase the risk of COVID-19 infection, 15 (14.7\%) of the patients answered were affirmative, and the most common drug used by these patients was meselasine.

During the pandemic, 83 (81.4\%) of the patients took their medications regularly. In 19 (18.6\%) patients with irregular drug usage, the most common reason for not being regular was the difficulty in accessing drugs (31.57\%), no specific reason (21.05\%), due to active infection (15.78\%), the belief that the drugs suppress the immune system (15.78\%), forgetfulness (15.78\%), and refusing to go to the hospital for infusion due to the risk of COVID-19 (5.26\%). Six (5.9\%) patients decreased their self-medication doses without consulting any physician, but did not discontinue it. Seven (36.8\%) of 19 people who did not consume the drugs regularly faced disease exacerbation in the last 3 months, and 3 (42.8\%) of these 7 subjects changed their medications without consulting any physician.

No significant difference was noted in terms of perceiving the risk of developing COVID-19 as a high risk between subjects who took or did not take regular medications and those who changed or did not change their medications on their own without consulting a physician. However, it was found that patients who changed their self-medication doses thought that they had a greater risk of acquiring COVID-19 ( $p=0.001)$.

In the last 3 months, 24 (17 UC and 7 CD, 23.5\%) patients experienced disease exacerbation and 14 patients (58.33\%) did not go to the hospital. COVID-19 infection (64.28\%) and not getting an appointment (21.42\%) were the most common reasons for not going to the hospital for treating the exacerbation. Considering 
the onset of symptoms and the duration of hospital admission, $4(40 \%)$ patients were admitted to the hospital immediately, 3 (30\%) patients were admitted within 1 week, and 3 (30\%) were admitted within 2-3 weeks (Table 3). Only 3 (12.5\%) patients who experienced disease exacerbation underwent an endoscopic procedure.

Cortisone treatment was initiated as follows: initial doses of cortisone in $6(25 \%)$ of the 24 patients who experienced an exacerbation during the pandemic; 3 (50\%) patients $20 \mathrm{mg} /$ day, 2 (33.3\%) patients $32 \mathrm{mg} /$ day, and 1 (16.7\%) patient 40 $\mathrm{mg} /$ day. In one patient, cortisone treatment was initiated before the pandemic and the dose reduction was started during pandemic period. In other words, 7 (6.9\%) patients received cortisone treatment in total. No COVID-19 infection was recorded in any of these patients during the follow-up period.

A total of 27 (26.4\%) IBD patients had researched the relationship between IBD and COVID-19 on the internet during the pandemic, and 12 (44.4\%) of them had sufficient information, while 10 (37.0\%) patients had insufficient information, and 5 (18.5\%) patients were unstable. No significant difference was noted between those who did research on the internet and those who did not in terms of the state of perceiving IBD disease as a risk for COVID-19 $(p>0.05)$. However, the rate of those who did research on the internet, the perception that their drug usage could increase their risk to COVID was significantly higher when compared to those who did not research ( $p=0.006)$. In addition, 96 (94.1\%) of the patients requested regular visits by phone during the pandemic period, and 95 of them (93.1\%) stated that they thought it would be beneficial.

During the pandemic, 97 (95.08\%) of the IBD patients without COVID-19 were questioned in terms of the most common symptoms of COVID-19 infection, and there were 67 (69.7\%) patients who shared at least one of the symptoms. The most common symptoms were diarrhea (29.03\%) and abdominal pain (20.64\%). On excluding these most common symptoms of IBD during the re-evaluation, the next most common symptoms were sore throat, weakness, and cough (Table 4).
During the pandemic, COVID-19 infection was observed in 5 (4.90\%) patients, including 4 patients with UC (pancolitis, $\mathrm{n}=3$; distal type, $\mathrm{n}=1$ ) and one patient with $\mathrm{CD}$ (colonic). Of these patients, 3 (60.0\%) reported irregular drug use. Only one patient experienced an exacerbation during the pandemic period, but did not go to the hospital for the same. The other 4 patients showed no signs of exacerbation of IBD disease during the COVID-19 infection period. None of the patients had a history of cortisone use and endoscopic procedures during the pandemic. Two patients were using infliximab, one patient was using infliximab + azathiopurine, and two patients were using mesalazine. Infliximab and azathiopurine treatments were interrupted during the infection period, and 2 patients receiving mesalazine continued with their medications. Due to COVID-19 infection, 3 patients were followed up at the hospital and two patients at home. Two of the patients used plaquenil, another two patients used plaquenil, azithromycin, and oseltamivir, and one patient used a combination of plaquenil, azithromycin, oseltamivir, and favipravir. Intensive care admission and death were not reported for any of these patients. Infliximab and azathioprine were restarted after COVID-19 polymerase chain reaction tests turned negative. All of the patients requested phone visits during the pandemic and did not research their diseases on the internet. The most common symptom of these patients with COVID-19 infection was fatigue (80.0\%).

\section{DISCUSSION}

IBD is a chronic inflammatory disease with exacerbation and remission periods. IBD patients perceive a risk of acquiring COVID-19 infection due to the usage of drugs such as immunosuppressants, immunomodulators, and antitumor necrosis factor (TNF) that could induce and maintain remission. In addition, it is an understandable condition that these patients are worried that the infection may progress to become more severe in the presence of COVID-19 infection (7). While the common GIS symptoms during COVID-19 infection are diarrhea, nausea, vomiting, abdominal pain, and anorexia, an exacerbation of IBD disease can be

Table 2. The perception of risk of patients for COVID-19

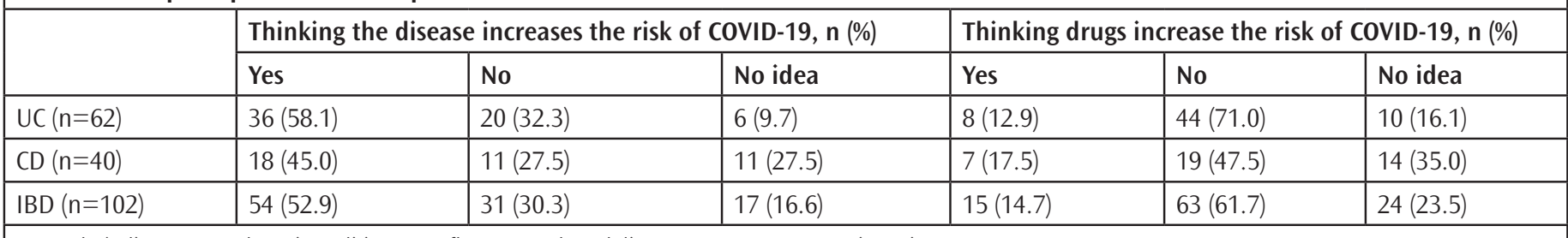

CD: Crohn's disease, UC: Ulcerative colitis, IBD: Inflammatory bowel disease, COVID-19: Coronavirus Disease-2019 
triggered during this course, which may further complicate the condition (1).

It is difficult to distinguish between the symptoms of GIS such as diarrhea, abdominal pain, nausea, and vomiting, which are common in IBD patients, due to IBD exacerbations or because they overlap with those of COVID-19 infection. In our study, when IBD patients without COVID-19 were questioned in terms of the common symptoms in COVID-19 infection, abdominal

\begin{tabular}{|c|c|}
\hline & n (\%) \\
\hline \multicolumn{2}{|l|}{ Disease exacerbation in the last 3 months } \\
\hline Yes & $24(23.5)$ \\
\hline No & $78(76.5)$ \\
\hline \multicolumn{2}{|l|}{ Going to the hospital after an exacerbation } \\
\hline Yes & $10(41.7)$ \\
\hline No & $14(58.3)$ \\
\hline \multicolumn{2}{|c|}{$\begin{array}{l}\text { The reason for having a disease exacerbation and not going to } \\
\text { the hospital }\end{array}$} \\
\hline Fear of risk of contracting COVID-19 & $9(64.2)$ \\
\hline Not being able to make an appointment & $3(21.4)$ \\
\hline Other & $2(14.3)$ \\
\hline \multicolumn{2}{|c|}{ Symptom onset and duration of hospitalization } \\
\hline At once & $4(40)$ \\
\hline Within 1 week & $3(30)$ \\
\hline Within 2-3 weeks & $3(30)$ \\
\hline After 3 weeks & $0(0)$ \\
\hline \multicolumn{2}{|c|}{ IBD: Inflammatory bowel disease, COVID-19: Coronavirus Disease-2019 } \\
\hline
\end{tabular}

\begin{tabular}{|l|l|l|}
\hline $\begin{array}{l}\text { Table 4. Symptom distribution of IBD patients without } \\
\text { COVID-19 infection }\end{array}$ \\
\hline Symptom type & $\begin{array}{l}\text { Number of } \\
\text { responses, } \mathbf{n}\end{array}$ & $\begin{array}{l}\text { Total } \\
\text { response, \% }\end{array}$ \\
\hline Diarrhea & 45 & 29.0 \\
\hline Abdominal pain & 32 & 20.6 \\
\hline Throat ache & 22 & 14.1 \\
\hline Cough & 16 & 10.3 \\
\hline Disappointment & 16 & 10.3 \\
\hline Fire & 12 & 7.7 \\
\hline Respiratory distress & 6 & 3.8 \\
\hline Myalgia & 3 & 1.9 \\
\hline Vomiting & 3 & 1.9 \\
\hline Headache & 0 & 0.0 \\
\hline Taste-odor disorder & 0 & 0.0 \\
\hline Total & 155 & 100 \\
\hline IBD: Inflammatory bowel disease, COVID-19: Coronavirus Disease-2019 \\
\hline
\end{tabular}

pain and diarrhea were the most common responses. Less rectal bleeding in COVID-19 patients is important in clinical history. While patients with COVID-19 may have leukopenia (especially lymphopenia) and thrombocytopenia, patients with IBD exacerbation can usually be distinguished by having normal lymphocyte and leukocyte values and normal or increased platelet values $(8,9)$. Diarrhea lasting for $>4$ weeks often suggests IBD-related colitis (10). In addition, the presence of symptoms such as cough, dyspnea, myalgia, contact with SARS-CoV-2positive patients, or a travel history from areas considered to be at high risk for COVID-19 infection in the past 14 days are also considered important for the differential diagnosis of COVID-19 infection $(11,12)$.

In more than half of the patients with COVID-19-induced pneumonia, stool samples have been shown to be positive for SARS-CoV-2 and the stool samples of one-fifth of all infected patients were positive for SARS-CoV-2 despite having negative respiratory samples $(13,14)$. Since inflammation in the intestines of IBD patients may increase the ACE2 expression, it can be assumed that patients with active IBD are an increased risk of SARS-CoV-2 infection. Although the delivery of SARS-CoV-2 occurs mainly through respiratory droplets, the ACE2 content is extremely low in the lungs than in other human tissues. This observation suggests that SARS-CoV-2 infection requires other not yet identified co-receptors or other additional factors than the ACE2 expression (15). In a study conducted for this purpose, IBD-associated inflammation alone was found to not fully meet the factors that allow the extra-respiratory passage of SARSCoV-2 (16). In addition, cytokines documented in patients with severe COVID-19 and those observed during the "cytokine storm" syndrome (such as IL-2, IL-6, TNF, and interferon) are similar to cytokines released from the inflamed intestine of patients with IBD. There are a few publications that report the use of medical therapies to suppress inflammation in IBD as a beneficial step to prevent pneumonia due to COVID-19 while reducing the ongoing mucosal inflammation (17-19). In our study, the frequency of COVID-19 infection among the followed-up IBD patients was found to be $4.9 \%$, none of which required intensive care and no mortality was reported. Four of these patients were receiving anti-TNF-based therapy.

Despite the presence of IBD being perceived as a potential risk for SARS-CoV-2, the limited available data and expert opinions suggest that IBD patients are not at an increased risk of SARSCoV-2 infection (20). However, in our study, more than half of the patients believed that their disease posed a risk for COVID-19 infection rather than their medications, and the most common 
drugs used by the patients who thought their drug usage was a risk factor was mesalazine. However, meselazines have very poor immunosuppressive effects and its long-term review is good safety side-effect profiles (21). The recommendations suggest that patients using mesalazine are at an increased risk to COVID-19 and hence their treatment should not be interrupted even during the active phase of the infection (22). In this regard, the use of both visual and written bulletins for IBD patients, addressing their concerns about the risks of drugs and their management should be well resolved.

During the pandemic, the use of regular medication is extremely important. Irregular drug use can cause severe disease flares, which may increase the need for additional treatment and endoscopic procedures. Considering that the primary priority of healthcare workers during the pandemic is COVID-19 and that they do not have spare time for IBD patients, it is recommended to not make changes in their medical treatments and to retain their current treatment regimens (23). In our study, approximately one-third of the patients using irregular medication experienced disease exacerbation and more than half of them could not go to the hospital due to the fear of infection or not getting an appointment. More than half of these patients who could go to the hospital were able to do so within 1-3 weeks of registering their complaints. This observation emphasizes the importance of the use of regular medications by patients during the pandemic.

The IBD population is generally composed of young patients. The relatively low prevalence of diseases such as diabetes, hypertension, and heart disease in this age group, which is associated with poor prognosis for COVID-19 infection, may explain the low comorbidity of COVID-19 infection despite that it is a chronic disease (24). Similarly, the mean age of the patients in the current study was $40.87 \pm 13.81$ years.

The frequency of COVID-19 was lower in patients treated with anti-TNF- $\alpha$ than in those who received steroids. It has been reported that only a few IBD patients with COVID-19 infection required hospitalization when treated with anti-TNF- $\alpha$ when compared to those who received the steroid therapy (25). COVID-19 infection was not observed among patients using steroids who were followed up. This could be due to the fact that, during the pandemic, patients with steroid treatment were informed about the risks related to infection and recommended with the general precautions; moreover, these patients were followed up via teleconference from home to avoid their traveling to the hospital as much as possible during the steroid treatment. As in our study, the International Organization for the Study of
IBD reported that telemedicine should be a fundamental part of patient management during the pandemic for IBD care, and this method is both cheaper and patient-oriented (26).

As is already known, during the pandemic, the general recommendations are that patients receiving treatment with anti-TNF- $\alpha$ and conical steroids should not discontinue their treatment if possible or, if the treatment has been planned but not started, other alternatives should be considered (27). In our study, in line with these recommendations, the received treatments by patients were not changed. Anti-TNF and azathioprine were suspended during the active COVID-19 infection period in IBD patients with COVID-19 treated with antiTNF- $\alpha$ only, and the treatment was restarted when the test came negative.

In a survey of IBD patients by Chen et al. (28), the respondents reported that they were concerned about the risk of infection and access to healthcare, and only a quarter of them remained in telemedicine communication with their follow-up doctors for IBD. In our study, more than half of the patients who experienced disease exacerbation for IBD did not go to the hospital for the fear of infection. In addition, 96 (94.1\%) of the patients requested regular visits via phone during the pandemic. It will thus be better to heed to these requests of the patients and design patient management plan accordingly in the following process.

\section{CONCLUSION}

Patients with IBD are those with chronic diseases that require immunosuppressive therapy. These patients should pay attention to the recommendations of the patient-based risk assessment of the prescribed drugs by the centers where they are followed so as to maintain the patients in their current treatment as much as possible and to take general protective measures to prevent infection. The majority of IBD patients with comorbidity should be treated and followed up via teleconference before requiring their visit to the hospital.

Action plans should be implemented in case of active IBD disease or COVID-19 infection. In addition, since this process is extremely dynamic, action plans supported by updated guidelines in the later stages of the pandemic will facilitate both physicians and patients to manage the process in an extremely healthy manner.

\section{Ethics}

Ethics Committee Approval: This study was approved by the Prof. Dr. Cemil Taşçığlu City Hospital/Local Human Research Ethics Committee, and all procedures conducted in the study involving human participants were in accordance with the ethical 
standards of the institutional and/or national research committee and with the 1964 Helsinki Declaration and its later amendments or comparable ethical standards. This study was approved by the Local Ethics Committee (12/05/2020 decision no: 156).

Informed Consent: All subjects included in this study agreed to their participation with their informed consent for the processing and collection of data for scientific purposes.

Peer-review: Externally peer-reviewed.

\section{Authorship Contributions}

Concept: Y.G., Design: S.A., Data Collection or Processing: M.A., Analysis or Interpretation: N.E., Literature Search: Y.G., N.E., Writing: N.E., H.S.

Conflict of Interest: No conflict of interest was declared by the authors.

Financial Disclosure: The authors declared that this study received no financial support.

\section{REFERENCES}

1. Samanta J, Dhar J, Khaliq A, Kochhar R. 2019 novel coronavirus infection: gastrointestinal manifestations. J Dig Endosc 2020;11:13.

2. Zhu N, Zhang D, Wang W. China Novel Coronavirus Investigating and Research Team. A novel coronavirus from patients with pneumonia in China, 2019. N Engl J Med 2020;382:727-33.

3. Hoffmann M, Kleine-Weber H, Schroeder S, Krüger N, Herrler T, Erichsen S, et al. SARS-CoV-2 cell entry depends on ACE2 and TMPRSS2 and is blocked by a clinically proven protease inhibitor. Cell 2020;181:271-80.

4. Xu H, Zhong L, Deng J, Peng J, Dan H, Zeng X, et al. High expression of ACE2 receptor of 2019-nCoV on the epithelial cells of oral mucosa. Int Oral Sci 2020;12:1-5.

5. Pan L, Mu M, Yang P, Sun Y, Wang R, Yan J, et al. Clinical characteristics of COVID-19 patients with digestive symptoms in Hubei. China: a descriptive, cross-sectional, multicenter study. Am J Gastroenterol 2020;115:766-73.

6. Hanauer SB. Inflammatory bowel disease: epidemiology, pathogenesis, and therapeutic opportunities. Inflamm Bowel Dis 2006;12(Suppl 1):S3-9.

7. Govani SM, Higgins PD. Combination of thiopurines and allopurinol: adverse events and clinical benefit in IBD. J Crohns Colitis 2010;4:444-9.

8. Alhazzani W, Møller M, Arabi Y, Loeb M, Gong M, Fan E, et al. Surviving sepsis campaign: guidelines on the management of critically ill adults with coronavirus disease 2019 (COVID-19). Intensive Care Med 2020;1-34.

9. Iacucci M, Cannatelli R, Labarile N, Mao R, Panaccione R, Danese S, et al. Endoscopy in inflammatory bowel diseases during the COVID-19 pandemic and post-pandemic period. Lancet Gastroenterol Hepatol 2020;5:598-606.

10. Fine KD, Schiller LR. AGA technical review on the evaluation and management of chronic diarrhea. Gastroenterology 1999;116:1464-86

11. Yang X, Yu Y, Xu J, Shu H, Liu H, Wu Y, et al. Clinical course and outcomes of critically ill patients with SARS-CoV-2 pneumonia in Wuhan, China: a single-centered, retrospective, observational study. Lancet Respir Med 2020;8:475-81.
12. Ying-Hui J, Lin C, Zhen-Shun C, Hong C, Tong D, Yi-Pin F, et al. A rapid advice guideline for the diagnosis and treatment of 2019 novel coronavirus (2019-nCoV) infected pneumonia (standard version). Mil Med Res 2020;7:4.

13. Wang W, Xu Y, Gao R, Lu R, Han K, Wu G, et al. Detection of SARS-CoV-2 in different types of clinical specimens. JAMA 2020;323:1843-4.

14. Xiao F, Tang M, Zheng X, Liu Y, Li X, Shan H. Evidence for gastrointestinal infection of SARS-CoV-2. Gastroenterology 2020;158:1831-3.

15. Hamming I, Timens W, Bulthuis M, Lely AT, Navis GJ, van Goor H. Tissue distribution of ACE2 protein, the functional receptor for SARS coronavirus. A first step in understanding SARS pathogenesis. J Pathol 2004;203:631-7.

16. Monteleone G, Franzè E, Laudisi F. Expression of receptors for SARSCoV-2 in the gut of patients with inflammatory bowel disease. Gut Liver 2020;14:530-1.

17. Chen C, Zhang X, Ju Z, He W. Advances in the research of cytokine storm mechanism induced by Corona Virus Disease 2019 and the corresponding immunotherapies. Zhonghua Shao Shang Za Zhi 2020;36:471-5

18. Maude SL, Barrett D, Teachey DT, Grupp SA. Managing cytokine release syndrome associated with novel T cell-engaging therapies. Cancer J 2014;20:119-22.

19. Monteleone G, Pallone F, MacDonald TT. Emerging immunological targets in inflammatory bowel disease. Curr Opin Pharmacol 2011;11:640-5

20. Monteleone G, Ardizzone S. Are patients with inflammatory bowel disease at increased risk for Covid-19 infection? J Crohns Colitis 2020;14:1334-6.

21. Ransford R, Langman M. Sulphasalazine and mesalazine: serious adverse reactions re-evaluated on the basis of suspected adverse reaction reports to the Committee on Safety of Medicines. Gut 2002;51:536-9.

22. Rubin DT, Feuerstein JD, Wang AY, Cohen RD. AGA clinical practice update on management of inflammatory bowel disease during the COVID-19 pandemic: expert commentary. Elsevier; 2020.

23. Kennedy NA, Jones G-R, Lamb CA, Appleby R, Arnott I, Beattie RM, et al. British Society of Gastroenterology guidance for management of inflammatory bowel disease during the COVID-19 pandemic. Gut 2020;69:984-90.

24. Allocca M, Fiorino G, Zallot C, Furfaro F, Gilardi D, Radice S, et al Incidence and patterns of COVID-19 among inflammatory bowel disease patients from the Nancy and Milan cohorts. Clin Gastroenterol Hepatol 2020;18:2134-5.

25. Brenner EJ, Ungaro RC, Colombel JF, Kappelman MD. IBD in the COVID-19 era: the value of international collaboration. Lancet Gastroenterol Hepatol 2020;5:887-8.

26. Lees CW, Regueiro M, Mahadevan U. Innovation in inflammatory bowel disease care during the COVID-19 pandemic: results of a global telemedicine survey by the International Organization for the Study of Inflammatory Bowel Disease. Gastroenterology 2020;159:805-8.

27. Tursi A, Vetrone LM, Papa A. Anti-TNF- $\alpha$ Agents in Inflammatory Bowe Disease and Course of COVID-19. Inflamm Bowel Dis 2020;26:e73.

28. Chen Y, Hu S, Wu H, Farraye FA, Bernstein CN, Zheng JJ, et al. Patterns of care for inflammatory bowel disease in China during the COVID-19 pandemic. Lancet Gastroenterol Hepatol 2020;5:632-4. 